\title{
Gene Therapy Progress and Prospects: Electroporation and other physical methods
}

\author{
DJ Wells \\ Gene Targeting Unit, Department of Cellular and Molecular Neuroscience, Division of Neuroscience and Psychological Medicine, \\ Imperial College London, Charing Cross Hospital, St Dunstan's Road, London, UK
}

Over the last 5 years, physical methods of plasmid delivery have revolutionized the efficiency of nonviral gene transfer, in some cases reaching the efficiencies of viral vectors. In vivo electroporation dramatically increases transfection efficiency for a variety of tissues. Other methods with clinical precedent, pressure-perfusion and ultrasound, also improve plasmid gene transfer. Alternatives such as focused laser, magnetic fields and ballistic (gene gun) approaches can also enhance delivery. As plasmid DNA appears to be a safe gene vector system, it seems likely that plasmid with physically enhanced delivery will be used increasingly in clinical trials.

Gene Therapy (2004) 11, 1363-1369. doi:10.1038/

sj.gt.3302337; Published online 5 August 2004

Keywords: plasmid DNA; gene transfer; ultrasound; hydrodynamic; vascular; skeletal muscle

In brief

\section{Progress}

- Plasmid-based gene transfer has advantages compared to the use of viral vectors.

- Application of an electrical field dramatically enhances plasmid gene transfer in vivo.

- Pressurized vascular delivery improves plasmid transfection in vivo with a more widespread distribution than local injection.

- Clinically applicable ultrasound can enhance plasmid based gene delivery in vivo.

- Other physical methods including laser, magnetic fields and ballistic delivery can also be used to increase in vivo gene transfer but are generally less effective.
Prospects

- Clinical precedents favour the use of electroporation, isolated limb/organ perfusion and ultrasound for future clinical trials involving gene transfer.

- Such physical methods are likely to be used first for treatment of local tumours and to enhance genetic vaccination.

- Possible trial of vascular pressure-mediated plasmid gene transfer for treatment of Duchenne muscular dystrophy.

- Physical methods may be used to enhance viral vector based gene delivery.

\section{Progress}

\section{Plasmid-based gene transfer has advantages compared to the use of viral vectors}

Recombinant viral vectors have been developed as highly efficient methods for gene delivery to a variety of tissues. The efficiency of these vectors is due to the presence of viral proteins that interact with cell surface receptors. Unfortunately, these viral proteins evoke specific immune responses that can limit the ability to re-administer the vector. This may be a particular problem in translation of preclinical studies to human

Correspondence: Dr DJ Wells, Gene Targeting Unit, Department of Cellular and Molecular Neuroscience, Division of Neuroscience and Psychological Medicine, Imperial College London, Charing Cross Hospital, St Dunstan's Road, London W6 8RP, UK

Published online 5 August 2004 treatments, as a significant proportion of the population may have previously encountered the virus and thus have neutralizing antibodies.

In contrast, plasmid vectors are composed entirely of covalently closed circles of double-stranded DNA with no associated proteins. However, gene delivery with plasmid vectors is highly inefficient unless the DNA is either associated with other molecules and/or physical energy is applied to aid cell entry. The use of liposomes and dendrimer complexes has enhanced gene delivery to some tissues in vivo, but the most important improvement has been the use of physical delivery systems that enable plasmid-based gene delivery to reach efficiencies close to that achieved with viral vectors.

Plasmid DNA, although itself a complex molecule, is substantially easier to mass-produce and quality control than are viral vectors. These issues, together with the low immunogenicity and lack of integration seen with 
plasmid DNA, make it a highly attractive reagent for human gene therapy provided that a high efficiency delivery can be achieved. Clinical trials to date using naked plasmid DNA have reported no ill effects. ${ }^{1,2}$

The physical methods shown to enhance plasmid transfection in vivo are reviewed below. All methods enhance gene transfer by bringing the plasmid DNA into closer proximity to the cell membrane and/or cause temporary microdisruption of the cell membrane, thus increasing the passage of plasmid into the cell.

\section{Application of an electrical field dramatically enhances plasmid gene transfer in vivo}

The most substantial change in the efficiency of plasmidbased gene transfer has been seen when delivery of plasmid is followed by the application of a series of electrical pulses as a form of in vivo electroporation (also referred to as electropermeabilization or electrotransfer). This technology is based on the earlier studies of in vitro electroporation and in vivo delivery of macromolecules to local tumours. The latter methodology has been successfully taken through to clinical trials with the cytotoxic drug bleomycin. ${ }^{3}$

In vivo electroporation of plasmid DNA was first used for skin and liver but skeletal muscle has recently attracted a lot of attention, as expression of the episomal plasmid can be long lived in this tissue. Indeed, a wide range of tissues have been studied including skin, kidney, lung, liver, skeletal and cardiac muscle, joints, spinal cord, brain, retina, cornea and the vasculature. ${ }^{4-7}$ In most studies, electroporation increased gene expression by 100 - to 1000 -fold compared to injection of naked plasmid DNA. The exact mechanism by which delivery of plasmid into cells is enhanced is not certain, although it is clear that membranes become effectively permeable once a critical voltage has been achieved (in the order of $200 \mathrm{~V} / \mathrm{cm}$ in vivo). This is thought to occur by the formation of hydrophilic pores and subsequent movement of plasmid through these pores as a local electrophoretic effect. A recent paper has challenged this widely held view. Golzio et $a l^{8}$ used fluorescently labelled plasmid to visualize the interaction with single cells in vitro. In their system, plasmid was seen to accumulate at the cell membrane via an electrophoretic effect but did not immediately move into the cytosol. Movement into the cytoplasm was relatively slow and continued after the application of the electrical field ended. This is consistent with the DNA active uptake mechanism proposed by Budker et al, ${ }^{9}$ but may represent a novel physical interaction with the cell membrane as, with time, the plasmid that had not yet entered the cytoplasm became inaccessible to a DNA dye. However, this plasmid accumulation has yet to be observed in vivo and the complex organization of tissues such as muscle may significantly modify this process. Very recently, Bureau et $a l^{10}$ reported the existence of a pool of plasmid that appears to escape the attention of extracellular nucleases and can be successfully electrotransfered up to $4 \mathrm{~h}$ after injection of the DNA.

The choice of electrode design is currently very variable and there is a clear need for good comparative studies. Zhang et $a l^{11}$ examined skin electroporation using a calliper electrode and compared this to a meander electrode (the latter being an array of inter- a
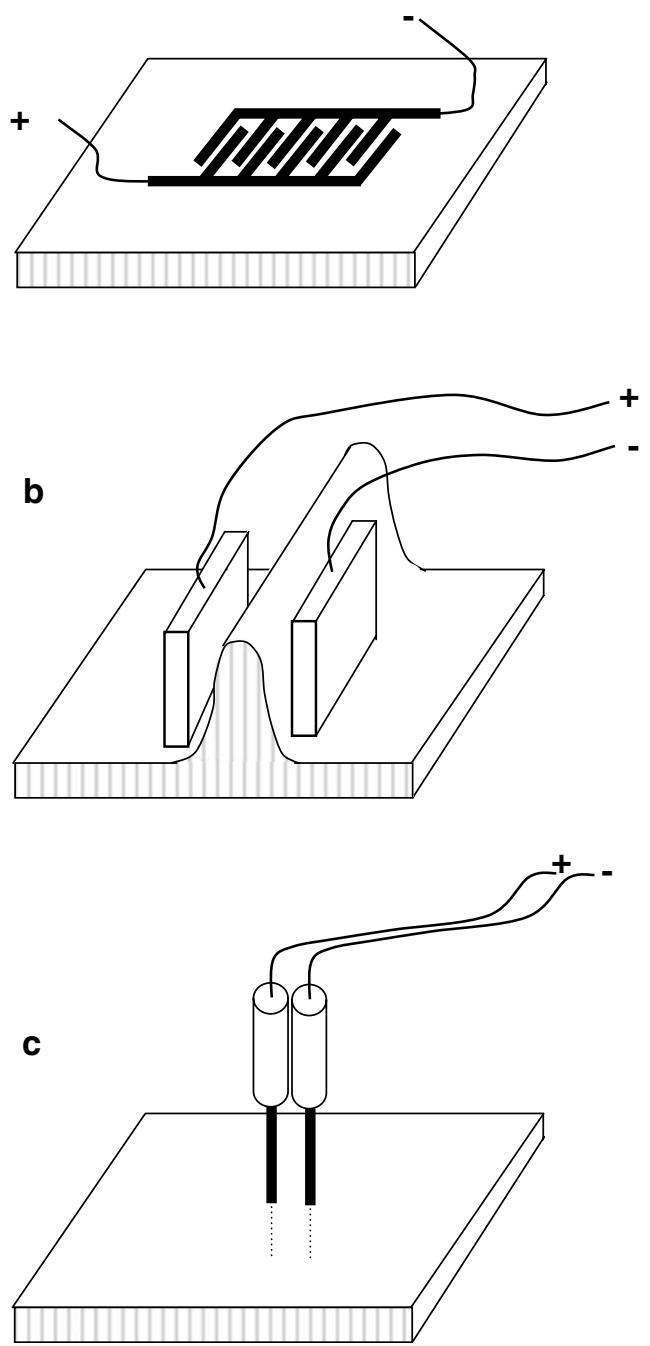

Figure 1 Meander (a), calliper (b) and needle (c) electrodes used for in vivo electrotransfer. In the case of the meander electrode, an electrical field is generated between each positive and negative electrode with part of the field entering the tissue. This electrode design is the least invasive of the three. With the calliper electrodes, a near-uniform electrical field is generated between the electrode plates. With needle electrodes, a field is generated between the needles. In some cases, multiple needle electrodes have been used.

locking positive and negative electrodes; Figure 1a). Both were equally effective but the meander electrode appears to be a more patient-friendly design as it avoids the need to pinch the skin between the callipers. For other tissues such as liver and muscle, there is a choice between plate and needle electrodes (Figure $1 b$ and $c$ ). In general, the plate electrodes appear to give a more uniform electrical field and are more commonly used for small animals studies but they may not be suitable for electrotransfer in large animals due to the large electrical fields that would need to be applied to larger tissues. The pattern of electrical pulses also varies considerably between studies ranging from moderate voltage (eg $200 \mathrm{~V} / \mathrm{cm}$ ) pulses of tens of milliseconds to high-voltage microsecond pulses. It is not yet clear what the optimal pattern is but Bureau et $a l^{12}$ showed that their best results in skeletal muscle were obtained when a single high-voltage pulse was 
followed by a series of low-voltage pulses. They proposed that the initial pulse effected an electroporation of the membrane and that the subsequent pulses electrophoresed the DNA into the muscle fibre. Currently, the majority of papers appear to use a pattern averaging $6-10$ pulses of $20-40 \mathrm{~ms}$ at $1 \mathrm{~Hz}$ with a field strength in the order of $200 \mathrm{~V} / \mathrm{cm}$.

One of the limitations of the electrotransfer is that there can be substantial damage associated with the procedure and that this can limit the efficiency of transfection. ${ }^{13} \mathrm{~A}$ recent paper by Durieux et al ${ }^{14}$ convincingly demonstrates that muscle damage is closely associated with the presence of the plasmid DNA during the electrotransfer and is made worse when the LacZ reporter gene is expressed. Modification of the magnitude and duration of the electrical pulses can ameliorate but not entirely prevent all the plasmid-associated damage following treatment of skeletal muscle.

Dean et $a l^{15}$ have recently provided a dramatic demonstration of the potential of in vivo electroporation for the lung. They applied calliper electrodes either side of the thorax of mice after intratracheal instillation of a solution of plasmid DNA. They showed substantial short-term reporter gene expression in the lung following this procedure with low mortality in the treated animals. Gene expression was seen predominantly in the peripheral alveoli and in both the epithelium and deeper cells. Histological analysis of the lungs $24 \mathrm{~h}$ after treatment did not reveal any obvious signs of damage. Thus electroporation appears a relatively safe procedure in the lungs. Application to humans might be possible using bronchoscope-directed electrodes but would only result in treatment of local areas of the lung.

Other recently developed targets for electroporation include joints, ${ }^{16}$ spinal cord $^{17}$ and the retina. ${ }^{18}$ Thus, Matsuda and Cepko ${ }^{19}$ have reported that very high gene transfer efficiencies into retinal cells, in particular the photoreceptors, can be achieved with electroporation in neonatal rats and mice. We have demonstrated that electroporation can also be used to deliver oligonucleotides to muscle, specifically for antisense-mediated exon skipping in dystrophic skeletal muscle. ${ }^{20}$

Electroporation has been extensively tested for gene transfer into tumours. Even the transfer of empty plasmid vector is sufficient to cause significant tumour regression in some models. ${ }^{21}$ Electroporation of plasmids containing interleukin 12 (IL-12) and interleukin 18 (IL18) has been shown to produce a synergistic effect in inhibiting tumour growth, both for the treated tumour and also for the untreated contralateral tumour. ${ }^{22}$ In another study, electrotransfer of a combination of bleomycin and a plasmid encoding IL-12 not only produced complete remission in a proportion of mice carrying a subcutaneous melanoma but also provided a significant protective effect against established metastases. ${ }^{23}$ Given the success of electrotransfer of bleomycin in many clinical trials, ${ }^{3}$ the combination with gene transfer appears to offer further potential in the delivery of a clinically effective treatment.

Liu and Huang ${ }^{24}$ have used a combination of vascular delivery with electroporation for the liver. Intravenous administration of plasmid via the tail vein in the mouse enhanced gene expression in the liver following electroporation compared to direct injection into the liver before electroporation. Expression was further improved by temporarily blocking blood flow through the vena cava or the portal vein and hepatic vein. It remains to be seen if this combination approach will work in other tissues.

Electrotransfer can also be used for genetic vaccination, and a number of laboratories have demonstrated substantial improvements in responses to a variety of antigens. ${ }^{25-27}$ Tjelle et $a l^{28}$ have proposed that electrotransfer of plasmid DNA encoding immunoglobulins might be an alternative to the administration of monoclonal antibodies in the treatment of a variety of conditions and have demonstrated prolonged expression in both mice and sheep.

An interesting development of in vivo electroporation for research purposes has been the application of this technology to the developing central nervous system (CNS) of rodent embryos. ${ }^{29-31}$ Using very fine electrodes, it is possible to deliver plasmids to defined regions of the developing brain to track cell migration and to modify patterns of development. Recently, Wei et $a l^{32}$ have extended this technology to adult rodents, showing that it is possible to express selectively plasmid-based constructs in specific regions of the adult CNS.

Finally, integration of plasmid DNA has not been previously reported following direct intramuscular injection despite a number of careful studies from several laboratories. In contrast, Wang et $a l^{33}$ have recently reported that integration of plasmid DNA can be detected following electroporation of skeletal muscle in vivo. However, this is still a very rare event, below the level of background genomic mutation, and so is unlikely to be a significant safety risk with respect to clinical application.

\section{Pressurized vascular delivery improves plasmid transfection in vivo with a more widespread distribution than local injection}

As plasmid DNA contains no proteins for attachment to cellular receptors, naked plasmid has no specific targeting to different cell types and thus it is essential that the DNA is placed in close contact with the desired cell type. The use of pressure to achieve this close contact has also dramatically increased the efficiency of delivery, in particular to liver and skeletal muscle.

The use of rapidly delivered extremely high volume (equivalent to total blood volume) injections via the tail vein of the mouse leads to highly efficient transfection of hepatocytes throughout the majority of the liver. A series of very recent papers have attempted to explain the physical basis for this phenomenon. Zhang et al ${ }^{34}$ have demonstrated that hydrodynamic gene delivery leads to a transient decrease in heart function and a rapid rise in venous pressure that leads to enlargement of the fenestrae in the liver sinusoids. This in turn allows hydrostatic pressure to act on the hepatocyte cell membranes causing transient pores or defects, a process they term hydroporation. Other groups ${ }^{35-37}$ have demonstrated that the dynamics of the transfection process support this concept of transient hyperpermeability of the hepatocyte cell membrane and do not support the active uptake mechanism proposed by Budker et al. ${ }^{9}$ The use of this hydrodynamic technique in the mouse has allowed comparison of the effect of different vector sequences, $^{38}$ the testing of optimal expression constructs $^{39}$ and the efficient delivery of siRNA to the 
liver. $^{40,41}$ The same technique has been applied to rats. ${ }^{42}$ However, this approach, while useful for studies in rodents, is not directly applicable to humans. Eastman et $\mathrm{al}^{43}$ examined local hydrodynamic delivery into specific lobes of the rabbit liver using balloon cuff catheters. They demonstrated that pressure-mediated local delivery can be safely achieved with this approach without the need for surgery to expose the liver. As with the tail-vein injections, there were only transient elevations in liverspecific enzymes released into the blood. Pressuremediated plasmid delivery has also been used for other tissues, such as the kidney. ${ }^{44}$ The importance of local hydrostatic pressure has been shown by the work of Liu et $a l^{45}$ who report that mechanical massage of the liver increases gene transfer following intravenous delivery of plasmid DNA.

Huang et $a l^{46}$ have also successfully delivered plasmid to the diaphragm using the venous approach with temporary occlusion of the cranial vena cava. For delivery to limb musculature, pressure-mediated plasmid delivery is generally performed via the arterial system. This approach has been used for delivery of plasmid to multiple muscles in the temporarily isolated limbs of primates, ${ }^{47}$ and isolated limb perfusion is an established technique in clinical practice for the treatment of tumours. ${ }^{48}$ Plasmid uptake and expression was more efficient than for direct intramuscular injection and was detected in all muscles of the perfused limb. There have been no subsequent publications using this gene delivery methodology, although unpublished studies were reported in the review by Herweijer and Wolff. ${ }^{49}$ We have observed expression of human minidystrophin (using a species-specific antibody) in up to $30 \%$ of muscle fibres in the limb muscles of rats following pressure-mediated arterial delivery (Fletcher, Wells and Wells, unpublished results). The same technique is currently being used to deliver dystrophin to dystrophic muscle in the legs of the canine model of X-linked muscular dystrophy. ${ }^{50}$ A very recent paper from Huang's group ${ }^{51}$ has shown that it may not be necessary to use the arterial route to deliver plasmid to skeletal muscle. They demonstrated remarkably high efficiency delivery of a dystrophin plasmid to dystrophic mouse muscle by both arterial and venous routes provided that there was a temporary occlusion of the blood supply that increased pressure within the muscle vascular bed.

\section{Clinically applicable ultrasound can enhance plasmid-based gene delivery in vivo}

In vivo electroporation- and pressure-mediated delivery, although very efficient, are rather invasive techniques. Consequently, other methods have been examined for their ability to provide motive force in the delivery of plasmid DNA. Ultrasound is in general clinical use for both therapeutic and diagnostic purposes. Low-level ultrasound is used for diagnostic imaging, ultrasonic shock waves are used in the treatment of kidney stones (lithotripter) and high-intensity focused ultrasound (HIFU) is used for the thermal destruction of tumours. A number of groups have demonstrated the utility of these various modalities of ultrasound in enhancing the delivery of plasmid DNA. For example, Taniyama et al have used low-dose ( $1 \mathrm{~min}$ at $1 \mathrm{MHz}, 2.5 \mathrm{~W} / \mathrm{cm}^{2}$ ) ultrasound to improve gene delivery in skeletal muscle $\mathrm{e}^{52}$ and the carotid artery. ${ }^{53}$ Likewise, other groups have used a similar approach for gene transfer into skeletal muscle ${ }^{54-56}$ and the heart. ${ }^{57,58}$ In contrast, Huber et al ${ }^{59}$ used HIFU ( $1 \mathrm{~min}$ at $0.85 \mathrm{MHz}, 155 \mathrm{~W}$ over a $2 \mathrm{~mm}$ width) to perform gene transfer to the carotid artery on the grounds that this modality allows specific localization of the enhanced gene transfer. They demonstrated an eight-fold increase in total reporter gene expression with HIFU alone and a 17.5-fold increase when ultrasound contrast bubbles were used. However, the reported increase in transfection with contrast enhanced HIFU is not significantly different from the fold increases shown in many of the lower power delivery ultrasound studies. HIFU and lithotripter shock waves have been used to simultaneously ablate tumours and perform gene transfer into the residual mass but gene expression was very variable, possibly due to variable degrees of tumour ablation. ${ }^{60}$

The application of ultrasound results in acoustic cavitation that can disrupt tissues and produce transient membrane permeabilization, thus enhancing the delivery of plasmid to the cytoplasm. Nucleation agents such as ultrasound contrast agents can enhance cavitation, and several studies have examined a range of such contrast agents, concluding that albumin-coated octa-fluoropropane gas microbubbles (Optison) is preferable to several other commonly available agents. ${ }^{61,62}$ Taniyama et al ${ }^{52}$ have shown electron micrographs of cells treated with Optison and ultrasound in culture that suggest that transient pores in the cell membrane are indeed opened up immediately following treatment. Even with the use of ultrasound contrast reagents, most studies only show an average of 10- to 15-fold increase in reporter gene expression, which is considerably below that which can be achieved with electroporation.

A combination of electroporation and ultrasound (electro-sonoporation) has been described by Yamashita et al. ${ }^{63}$ In their study, electro-sonoporation was superior to either electroporation or ultrasound alone. However, it is difficult to compare this study to others in the field due to insufficient methodological details for the electroporation component.

\section{Other physical methods such as laser, magnetic fields and ballistic delivery can also be used to increase in vivo gene transfer but are generally less effective}

Focused laser provides an alternative energy source, and the potential of this technique was demonstrated in a recent paper by Zeira et al. ${ }^{64}$ The authors reported that gene delivery into muscle could be enhanced by application of a femtosecond infrared laser (5 s at $30 \mathrm{~mW}$ ) and they compared this to in vivo electroporation of the same quantity of DNA into the same muscle $(16 \times 20 \mathrm{~ms}$ pulses at $200 \mathrm{~V} / \mathrm{cm})$. The authors concluded that there was a similar efficiency of gene delivery and that this was accompanied by substantially less damage when using laser beam gene transduction (LBGT). The mechanism by which the laser enhanced gene transfer was not clear but likely involved local disruption of the muscle cell membrane. Two issues arise from this work. The comparison with electroporation was not unexpected as several other groups, including ourselves, have shown that multiple pulses at $200 \mathrm{~V} / \mathrm{cm}$ cause substantial muscle damage and that modifications to the 
protocol can reduce this damage. ${ }^{13,65}$ Secondly, the laser was focused to $2 \mathrm{~mm}$ under the skin whereas substantially deeper focusing would be required for percutaneous delivery to human skeletal muscle. It remains to be seen if LBGT can be significantly scaled up in studies of larger muscles or other tissues.

Strong magnetic fields can also be used to provide an energy source to assist gene transfer when plasmid DNA is coupled to paramagnetic nanoparticles. Application of magnetic fields following local injection of plasmidassociated nanoparticles (magnetofection) has been reported to enhance in vivo gene transfer to the gastrointestinal tract and the vasculature of the ear. ${ }^{66}$ The improved gene transfer associated with magnetofection does not appear to be as substantial as that seen with hydrodynamic delivery or electroporation, although direct comparisons have not yet been made.

Bombardment of tissues by plasmid-coated microparticles has previously received considerable attention for gene transfer in animals after originally being developed for gene delivery to plants. The application of the gene gun technology in animals has been limited to superficial tissues such as the skin. Recent papers have continued to use this approach for applications such as gene therapy of bladder pain using pro-opiomelanocortin gene transfer in a rat model, ${ }^{67}$ the transfer of IL-10, IL-12 and B7.1 into murine tumors, ${ }^{68}$ gene transfer into the heart, ${ }^{69}$ gene transfer into mouse embryos ${ }^{70}$ and treatment via the skin of a mouse model of lysosomal acid maltase deficiency (GAA, also known as glycogen storage disease type II). ${ }^{71}$ Most significantly, Dileo et $a l^{72}$ have recently reported a new design of gene gun that delivers microparticles at a higher pressure, thus accessing subcutaneous tissues, such as muscle or tumours, and consequently achieving longer-term gene expression.

Ballistic gene delivery has been used most extensively for the delivery of DNA vaccines to the skin. This approach has been reported to be superior to other methods of plasmid delivery to the $\operatorname{skin}^{73,74}$ but tends to generate a Th2 (humoral) rather than the Th1 (cytotoxic) immune response that commonly follows intramuscular administration. ${ }^{75,76}$ This difference in the nature of the immune response can be modified by different boost strategies following the initial priming vaccination by gene gun delivery of plasmid DNA. ${ }^{77}$

\section{Prospects}

\section{Application of physical methods for human gene therapy}

The dramatic improvements in plasmid gene transfer and expression achieved by the variety of physical methods reviewed above have transformed the use of plasmid vectors as a laboratory tool for answering both basic and applied research questions. Although there are clinical precedents for the use of electroporation, isolated limb/organ perfusion and ultrasound, it still remains to be seen if any of these approaches will be translated into gene therapy clinical trials. The most likely areas of application will be for local administration to tumours and for enhanced genetic vaccination. Previous genetic vaccination studies using naked plasmid DNA have been disappointing. ${ }^{78}$ However, electrotransfer into mouse muscle can produce a more dramatic induction of CD8 $\mathrm{T}$ cells than any other form of vaccination including the use of live vaccinia virus. ${ }^{79}$ Importantly, the use of electroporation maintains a high efficiency of vaccination when pre-clinical studies are moved from small to large animal models. ${ }^{80}$

The recent Phase 1 clinical trial studying the safety of plasmid-based gene transfer of dystrophin in Duchenne and Becker muscular dystrophy patients in France has been completed and initial reports confirm that no safety problems were encountered, although as expected the efficiency of gene delivery using direct intramuscular injection was very low compared to similar studies in dystrophic mice. Studies of arterial pressure-mediated gene transfer in dystrophic dogs show promising results and it is hoped that the same technique but involving an individual muscle will be the subject of the next clinical trial. ${ }^{50} \mathrm{~A}$ similar method has also been used to deliver adeno-associated viral vectors to multiple muscles in the haemophiliac $\operatorname{dog}^{81}$ and the use of physical delivery methods may also prove useful for viral as well as nonviral vectors.

Physical enhancement of viral gene delivery has been less widely investigated compared to the delivery of nonviral vectors. Emani et $a l^{82}$ have reported that optimal coronary artery delivery of adenoviral vectors requires pressures greater than normal systolic but that the threshold for this effect is limited and trebling the pressure induces marked cardiac damage. Similarly, high pressure was used to deliver an adenoviral vector via a double balloon catheter in the rat coronary artery. ${ }^{83}$ Ultrasound has also been used to deliver adenoviral vectors to the heart and was dependent on the presence of liposome microbubbles. ${ }^{84}$ Indeed, ultrasound may be used more in the future for viral vectors where it is desirable to target specific tissues.

\section{References}

1 Comerota AJ et al. Naked plasmid DNA encoding fibroblast growth factor type 1 for the treatment of end-stage unreconstructible lower extremity ischemia: preliminary results of a phase I trial. J Vasc Surg 2002; 35: 930-936.

2 Losordo DW et al. Phase 1/2 placebo-controlled, double-blind, dose-escalating trial of myocardial vascular endothelial growth factor 2 gene transfer by catheter delivery in patients with chronic myocardial ischemia. Circulation 2002; 105: 2012-2018.

3 Gothelf A, Mir LM, Gehl J. Electrochemotherapy: results of cancer treatment using enhanced delivery of bleomycin by electroporation. Cancer Treat Rev 2003; 29: 371-387.

4 Bigey $\mathrm{P}$, Bureau MF, Scherman D. In vivo plasmid DNA electrotransfer. Curr Opin Biotechnol 2002; 13: 443-447.

5 Gehl J. Electroporation: theory and methods, perspectives for drug delivery, gene therapy and research. Acta Physiol Scand 2003; 177: 437-447

6 McMahon JM, Wells DJ. Electroporation for gene transfer to skeletal muscles: current status. BioDrugs 2004; 18: 155-165.

7 Bloquel C, Fabre E, Bureau MF, Scherman D. Plasmid DNA electrotransfer for intracellular and secreted proteins expression: new methodological developments and applications. J Gene Med 2004; 6 (Suppl 1): S11-S23.

8 Golzio M, Teissie J, Rols MP. Direct visualization at the singlecell level of electrically mediated gene delivery. Proc Natl Acad Sci USA 2002; 99: 1292-1297. 
9 Budker V et al. Hypothesis: naked plasmid DNA is taken up by cells in vivo by a receptor-mediated process. J Gene Med 2000; 2: $76-88$.

10 Bureau MF et al. Intramuscular plasmid DNA electrotransfer: biodistribution and degradation. Biochim Biophys Acta 2004; 1676: 138-148.

11 Zhang L, Nolan E, Kreitschitz S, Rabussay DP. Enhanced delivery of naked DNA to the skin by non-invasive in vivo electroporation. Biochim Biophys Acta 2002; 1572: 1-9.

12 Bureau MF et al. Importance of association between permeabilization and electrophoretic forces for intramuscular DNA electrotransfer. Biochim Biophys Acta 2000; 1474: 353-359.

13 McMahon JM et al. Optimisation of electrotransfer of plasmid into skeletal muscle by pretreatment with hyaluronidase increased expression with reduced muscle damage. Gene Therapy 2001; 8: 1264-1270.

14 Durieux AC, Bonnefoy R, Busso T, Freyssenet D. In vivo gene electrotransfer into skeletal muscle: effects of plasmid DNA on the occurrence and extent of muscle damage. J Gene Med 2004; 6: 809-816.

15 Dean DA et al. Electroporation as a method for high-level nonviral gene transfer to the lung. Gene Therapy 2003; 10: 1608-1615.

16 Ohashi $\mathrm{S}$ et al. Successful genetic transduction in vivo into synovium by means of electroporation. Biochem Biophys Res Commun 2002; 293: 1530-1535.

17 Lin CR et al. Electroporation for direct spinal gene transfer in rats. Neurosci Lett 2002; 317: 1-4.

18 Dezawa $\mathrm{M}$ et al. Gene transfer into retinal ganglion cells by in vivo electroporation: a new approach. Micron 2002; 33: 1-6.

19 Matsuda T, Cepko CL. Electroporation and RNA interference in the rodent retina in vivo and in vitro. Proc Natl Acad Sci USA 2004; 101: 16-22.

20 Wells KE et al. Enhanced in vivo delivery of antisense oligonucleotides to restore dystrophin expression in adult $\mathrm{mdx}$ mouse muscle. FEBS Lett 2003; 552: 145-149.

21 Heller LC, Coppola D. Electrically mediated delivery of vector plasmid DNA elicits an antitumor effect. Gene Therapy 2002; 9: 1321-1325.

22 Tamura T et al. Combination of IL-12 and IL-18 of electro-gene therapy synergistically inhibits tumor growth. Anticancer Res 2003; 23: 1173-1179.

23 Kishida $\mathrm{T}$ et al. Electrochemo-gene therapy of cancer: intratumoral delivery of interleukin-12 gene and bleomycin synergistically induced therapeutic immunity and suppressed subcutaneous and metastatic melanomas in mice. Mol Ther 2003; 8: 738-745.

24 Liu F, Huang L. Electric gene transfer to the liver following systemic administration of plasmid DNA. Gene Therapy 2002; 9: 1116-1119.

25 Tollefsen $S$ et al. Improved cellular and humoral immune responses against Mycobacterium tuberculosis antigens after intramuscular DNA immunisation combined with muscle electroporation. Vaccine 2002; 20: 3370-3378.

26 Zhang $\mathrm{L}$ et al. Accelerated immune response to DNA vaccines. DNA Cell Biol 2003; 22: 815-822.

27 Scheerlinck JP et al. In vivo electroporation improves immune responses to DNA vaccination in sheep. Vaccine 2004; 22: 1820-1825.

28 Tjelle TE et al. Monoclonal antibodies produced by muscle after plasmid injection and electroporation. Mol Ther 2004; 9: 328-336.

29 Fukuchi-Shimogori T, Grove EA. Neocortex patterning by the secreted signaling molecule FGF8. Science 2001; 294: 1071-1074.

30 Saito T, Nakatsuji N. Efficient gene transfer into the embryonic mouse brain using in vivo electroporation. Dev Biol 2001; 240: 237-246.

31 Tabata H, Nakajima K. Efficient in utero gene transfer system to the developing mouse brain using electroporation: visualization of neuronal migration in the developing cortex. Neuroscience 2001; 103: 865-872.

32 Wei $\mathrm{F}$ et al. Calmodulin regulates synaptic plasticity in the anterior cingulate cortex and behavioral responses: a microelectroporation study in adult rodents. J Neurosci 2003; 23: 8402-8409.

33 Wang Z et al. Detection of integration of plasmid DNA into host genomic DNA following intramuscular injection and electroporation. Gene Therapy 2004; 11: 711-721.

34 Zhang $G$ et al. Hydroporation as the mechanism of hydrodynamic delivery. Gene Therapy 2004; 11: 675-682.

35 Andrianaivo $\mathrm{F}$ et al. Hydrodynamics-based transfection of the liver: entrance into hepatocytes of DNA that causes expression takes place very early after injection. J Gene Med 2004, online in advance of print.

36 Kobayashi $\mathrm{N}$ et al. Hepatic delivery of particulates in the submicron range by a hydrodynamics-based procedure: implications for particulate gene delivery systems. J Gene Med 2004; 6: 455-463.

37 Kobayashi N, Nishikawa M, Hirata K, Takakura Y. Hydrodynamics-based procedure involves transient hyperpermeability in the hepatic cellular membrane: implication of a nonspecific process in efficient intracellular gene delivery. J Gene Med 2004; 6: 584-592.

38 Ehrhardt A et al. Optimization of cis-acting elements for gene expression from nonviral vectors in vivo. Hum Gene Ther 2003; 14: 215-225.

39 Alino SF, Crespo A, Dasi F. Long-term therapeutic levels of human alpha-1 antitrypsin in plasma after hydrodynamic injection of nonviral DNA. Gene Therapy 2003; 10: 1672-1679.

40 Lewis DL et al. Efficient delivery of siRNA for inhibition of gene expression in postnatal mice. Nat Genet 2002; 32: 107-108.

41 McCaffrey AP et al. RNA interference in adult mice. Nature 2002; 418: 38-39.

42 Maruyama $\mathrm{H}$ et al. High-level expression of naked DNA delivered to rat liver via tail vein injection. J Gene Med 2002; 4: 333-341.

43 Eastman SJ et al. Development of catheter-based procedures for transducing the isolated rabbit liver with plasmid DNA. Hum Gene Ther 2002; 13: 2065-2077.

44 Maruyama $\mathrm{H}$ et al. Kidney-targeted naked DNA transfer by retrograde renal vein injection in rats. Hum Gene Ther 2002; 13: 455-468.

45 Liu F, Lei J, Vollmer R, Huang L. Mechanism of liver gene transfer by mechanical massage. Mol Ther 2004; 9: 452-457.

46 Liu F, Nishikawa M, Clemens PR, Huang L. Transfer of fulllength Dmd to the diaphragm muscle of $\mathrm{Dmd}(\mathrm{mdx} / \mathrm{mdx})$ mice through systemic administration of plasmid DNA. Mol Ther 2001; 4: 45-51.

47 Zhang $\mathrm{G}$ et al. Efficient expression of naked DNA delivered intraarterially to limb muscles of nonhuman primates. Hum Gene Ther 2001; 12: 427-438.

48 Eggermont AM, de Wilt JH, ten Hagen TL. Current uses of isolated limb perfusion in the clinic and a model system for new strategies. Lancet Oncol 2003; 4: 429-437.

49 Herweijer H, Wolff JA. Progress and prospects: naked DNA gene transfer and therapy. Gene Therapy 2003; 10: 453-458.

50 Braun S. Duchenne muscular dystrophy gene therapy. Abstract Inv 26, ESGT 11th Annual Congress, November 14-17, 2003.

51 Liang KW et al. Restoration of dystrophin expression in $\mathrm{mdx}$ mice by intravascular injection of naked DNA containing fulllength dystrophin cDNA. Gene Ther 2004; 11: 901-908.

52 Taniyama $\mathrm{Y}$ et al. Development of safe and efficient novel nonviral gene transfer using ultrasound: enhancement of transfection efficiency of naked plasmid DNA in skeletal muscle. Gene Therapy 2002; 9: 372-380.

53 Taniyama Y et al. Local delivery of plasmid DNA into rat carotid artery using ultrasound. Circulation 2002; 105: 1233-1239. 
54 Schratzberger $\mathrm{P}$ et al. Transcutaneous ultrasound augments naked DNA transfection of skeletal muscle. Mol Ther 2002; 6: 576-583.

55 Danialou G et al. Ultrasound increases plasmid-mediated gene transfer to dystrophic muscles without collateral damage. $\mathrm{Mol}$ Ther 2002; 6: 687-693.

$56 \mathrm{Lu}$ QL, Liang HD, Partridge T, Blomley MJ. Microbubble ultrasound improves the efficiency of gene transduction in skeletal muscle in vivo with reduced tissue damage. Gene Therapy 2003; 10: 396-405.

57 Chen $S$ et al. Optimization of ultrasound parameters for cardiac gene delivery of adenoviral or plasmid deoxyribonucleic acid by ultrasound-targeted microbubble destruction. J Am Coll Cardiol 2003; 42: 301-308.

58 Bekeredjian $\mathrm{R}$ et al. Ultrasound-targeted microbubble destruction can repeatedly direct highly specific plasmid expression to the heart. Circulation 2003; 108: 1022-1026.

59 Huber PE et al. Focused ultrasound (HIFU) induces localized enhancement of reporter gene expression in rabbit carotid artery. Gene Therapy 2003; 10: 1600-1607.

60 Miller DL, Song J. Tumor growth reduction and DNA transfer by cavitation-enhanced high-intensity focused ultrasound in vivo. Ultrasound Med Biol 2003; 29: 887-893.

61 Li T, Tachibana K, Kuroki M. Gene transfer with echo-enhanced contrast agents: comparison between Albunex, Optison, and Levovist in mice - initial results. Radiology 2003; 229: 423-428.

62 Pislaru SV et al. Optimization of ultrasound-mediated gene transfer: comparison of contrast agents and ultrasound modalities. Eur Heart J 2003; 24: 1690-1698.

63 Yamashita $\mathrm{Y}$ et al. In vivo gene transfer into muscle via electrosonoporation. Hum Gene Ther 2002; 13: 2079-2084.

64 Zeira E et al. Femtosecond infrared laser - an efficient and safe in vivo gene delivery system for prolonged expression. Mol Ther 2003; 8: 342-350.

65 Durieux AC, Bonnefoy R, Manissolle C, Freyssenet D. Highefficiency gene electrotransfer into skeletal muscle: description and physiological applicability of a new pulse generator. Biochem Biophys Res Commun 2002; 296: 443-450.

66 Scherer F et al. Magnetofection: enhancing and targeting gene delivery by magnetic force in vitro and in vivo. Gene Therapy 2002; 9: 102-109.

67 Chuang YC et al. Gene therapy for bladder pain with gene gun particle encoding pro-opiomelanocortin cDNA. J Urol 2003; 170: 2044-2048.

68 Kitagawa $\mathrm{T}$ et al. Advantages and limitations of particlemediated transfection (gene gun) in cancer immuno-gene therapy using IL-10, IL-12 or B7-1 in murine tumor models. J Gene Med 2003; 5: 958-965.

69 Matsuno $\mathrm{Y}$ et al. Nonviral gene gun mediated transfer into the beating heart. ASAIO J 2003; 49: 641-644.

70 Yoshizawa $\mathrm{J}$ et al. Successful in utero gene transfer using a gene gun in midgestational mouse fetuses. J Pediatr Surg 2004; 39: 81-84.

71 Martiniuk $\mathrm{F}$ et al. Helios gene gun particle delivery for therapy of acid maltase deficiency. DNA Cell Biol 2002; 21: 717-725.

72 Dileo J, Miller Jr TE, Chesnoy S, Huang L. Gene transfer to subdermal tissues via a new gene gun design. Hum Gene Ther 2003; 14: 79-87.

73 Trimble $\mathrm{C}$ et al. Comparison of the CD8+ T cell responses and antitumor effects generated by DNA vaccine administered through gene gun, biojector, and syringe. Vaccine 2003; 21: 4036-4042.

74 Barfoed AM et al. Influence of routes and administration parameters on antibody response of pigs following DNA vaccination. Vaccine 2004; 22: 1395-1405.

75 Lima KM et al. Efficacy of DNA-hsp65 vaccination for tuberculosis varies with method of DNA introduction in vivo. Vaccine 2003; 22: 49-56.

76 Sasaki $S$ et al. Adjuvant formulations and delivery systems for DNA vaccines. Methods 2003; 31: 243-254.

77 Zhu W, Thomas CE, Sparling PF. DNA immunization of mice with a plasmid encoding Neisseria gonorrhoeae PorB protein by intramuscular injection and epidermal particle bombardment. Vaccine 2004; 22: 660-669.

78 Liu MA. DNA vaccines: a review. J Intern Med 2003; 253: 402-410.

79 Paster $\mathrm{W}$ et al. In vivo plasmid DNA electroporation generates exceptionally high levels of epitope-specific CD8+ T-cell responses. Gene Therapy 2003; 10: 717-724.

80 Babiuk $\mathrm{S}$ et al. Electroporation improves the efficacy of DNA vaccines in large animals. Vaccine 2002; 20: 3399-3408.

81 Arruda VR et al. Sustained correction of hemophilia B phenotype following intravascular delivery of AAV vector to skeletal muscle. Mol Ther 2002; 5: S157.

82 Emani SM et al. Catheter-based intracoronary myocardial adenoviral gene delivery: importance of intraluminal seal and infusion flow rate. Mol Ther 2003; 8: 306-313.

83 Ding $\mathrm{Z}$ et al. A minimally invasive approach for efficient gene delivery to rodent hearts. Gene Therapy 2004; 11: 260-265.

84 Chen $\mathrm{S}$ et al. Optimization of ultrasound parameters for cardiac gene delivery of adenoviral or plasmid deoxyribonucleic acid by ultrasound-targeted microbubble destruction. J Am Coll Cardiol 2003; 42: 301-308. 